\title{
GRB 970228 and GRB 980329 and the nature of their host galaxies
}

\author{
D.Q. Lamb, F.J. Castander, and D.E. Reichart \\ Department of Astronomy and Astrophysics, University of Chicago, 5640 South Ellis Avenue, Chicago, IL 60637, U.S.A.
}

Received January 21; accepted June 30, 1999

\begin{abstract}
We find that the local galactic extinction towards the field of gamma-ray burst GRB 970228 is $A_{V}=$ $1.09_{-0.20}^{+0.10}$, which implies a substantial dimming and change in the spectral slope of the intrinsic GRB 970228 afterglow. We measure a color $\left(V_{606}-I_{814}\right)_{\mathrm{ST}}=-0.18_{-0.61}^{+0.51}$ for the extended source coincident with the afterglow. Taking into account our measurement of the extinction toward this field, this color implies that the extended source is most likely a galaxy undergoing star formation, in agreement with our earlier conclusion (Castander \& Lamb 1998). In a separate analysis, we find that the inferred intrinsic spectrum of the GRB 980329 afterglow is consistent with the predictions of the simplest relativistic fireball model. We also find that the intrinsic spectrum of the afterglow is extincted both by dust (source frame $A_{V} \gtrsim$ $1 \mathrm{mag}$ ), and that the shape of the extinction curve is typical of young star-forming regions like the Orion Nebula but is not typical of older star-forming or starburst regions. The $\approx 2$ mag drop between the $R$ and the $I$ bands can be explained by the far-ultraviolet non-linear component of the extinction curve if $3 \lesssim z \lesssim 4$, and by the $2175 \AA$ bump if $z \approx 2$; other redshifts are not consistent with the observational data, given our general model.
\end{abstract}

Key words: gamma-rays: bursts

\section{Introduction}

The GRB 970228 and GRB 980329 afterglows are among the most well observed of the afterglows detected so far. We use observations of these afterglows to derive some of the properties of these bursts and to constrain the nature of their host galaxies.

\section{GRB 970228 and the nature of its host galaxy}

We have determined the local galactic extinction toward the GRB 970228 field by comparing galaxy counts in two bands in this field to those in the HDF, and by comparing the observed broad band colors of stars in the GRB 970228 field to the colors of library spectra of the same spectral type. We also estimate the extinction using the neutral hydrogen column density and the amount of infrared dust emission toward this field. Combining the results of these methods, we find a best-fit galactic extinction in the optical of $A_{V}=1.09_{-0.20}^{+0.10}$, which implies a substantial dimming and change of the spectral slope of the intrinsic

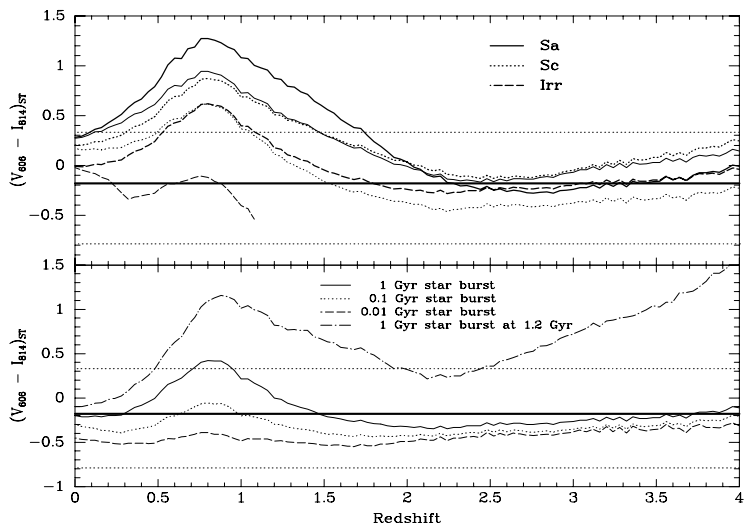

Fig. 1. WFPC2 colors for synthesized Spectral Energy Distributions (SEDs) versus redshift. The top panel shows the expected colors for spirals and irregular galaxies including a local extinction of $A_{V}=1.09$. The thick lines include a K-correction, the thin lines include evolutionary plus $\mathrm{K}$-corrections. The thick horizontal line is the best value observed color and the horizontal dotted lines its $1 \sigma$ error. The bottom panel shows models of 1 Gyr (solid line), $100 \mathrm{Myr}$ (dotted line) and $10 \mathrm{Myr}$ (dashed line) starbursts observed at redshift $z$. The dot-dashed line represents a 1 Gyr starburst observed at redshift $z, 200 \mathrm{Myr}$ after the cessation of star formation. The cessation of star formation, with the resulting disappearance of the most massive stars, produces redder colors incompatible with the observations

GRB 970228 afterglow. Further details can be found in Castander \& Lamb (1998, 1999a); see also González et al. (1999).

Re-analyzing the HST images, we measure a color $\left(V_{606}-I_{814}\right)_{\mathrm{ST}}=-0.18_{-0.61}^{+0.51}$ for the extended source. We constrain the nature of the likely host galaxy of GRB 970228 by comparing this color to those obtained from synthetic galaxy spectra computed with PEGASE (Fioc \& Rocca-Volmerange 1997), taking into account the measured extinction. The top panel of Fig. 1 shows the expected colors of an Sa, an Sc and an Irregular galaxy with and without evolution included. Galaxies are only consistent with the observed color if they are at high redshift $(z \gtrsim 1.0-1.5)$, or have active star formation (like the evolving Irr shown).

The bottom panel of Fig. 1 better illustrates this point. It shows that on-going bursts of star formation of duration shorter than $1 \mathrm{Gyr}$ produce acceptable $V_{606}-I_{814}$ 
colors; longer duration bursts are disfavored for redshifts $z \gtrsim 0.8$. If we include the $H$ and $K$ magnitudes of Fruchter et al. (1998) in our analysis, our conclusions are strengthened (see Castander \& Lamb 1999b for further details). We conclude that the host galaxy must be undergoing star formation, in agreement with our earlier result (Castander \& Lamb 1998; see also Castander \& Lamb 1999b). If there is any extinction present due to the host galaxy, this conclusion would be strengthened.

If the extended source is a galaxy with ongoing star formation, strong emission lines are expected. Tonry et al. (1997) and Kulkarni et al. (1997) have tried to obtain the spectrum of the GRB 970228 afterglow and its associated nebulosity. Neither observation revealed any obvious emission lines. The lack of observed [OII] and Ly $\alpha$ emission lines suggests that the redshift of the galaxy may lie in the range $1.5 \lesssim z \lesssim 2.6$, considering the spectral coverage of the observations.

\section{GRB 980329 and the nature of its host galaxy}

We model the observed radio through X-ray spectrum of the GRB 980329 afterglow, and its evolution through time, as follows. We take the intrinsic spectrum to be a thrice broken power law, motivated by the relativistic fireball model, in which spectral breaks may occur due to synchrotron self-absorption, the synchrotron peak, and electron cooling (see e.g., Sari et al. 1998). The spectrum that we fit is a generalization of the spectrum expected in this model, in the sense that we do not constrain the slopes of the four spectral segments, nor the (power-law) rates at which these segments fade, a priori.

We allow the intrinsic spectrum to be modified in the following ways. First, we allow this spectrum to be extincted by dust and absorbed by the Lyman limit at a single redshift, assumed to be the redshift of the burst and its host galaxy. We adopt the six parameter ultraviolet extinction curve of Fitzpatrick \& Massa (1988) and the one parameter optical and near-infrared extinction curve of Cardelli et al. (1989). Finally, we redshift the modified spectrum to the observer's frame of reference, and model the Lyman- $\alpha$ forest due to absorption by gas clouds along the line-of-sight between the burst and the observer.

The afterglow of GRB 980329 is unique among afterglows observed to date in that enough measurements of it have been taken to determine all the parameters of our model. From the results of our fits, we draw six conclusions: (1) The inferred intrinsic spectrum of the afterglow is consistent with the predictions of the simplest relativistic fireball model, in which an isotropic fireball expands into a homogeneous external medium. (2) The intrinsic spectrum of the afterglow is extincted by dust (source frame $A_{V} \gtrsim 1 \mathrm{mag}$ ). (3) The linear component of the extinction curve is flat, which is typical of young starforming regions like the Orion Nebula but is not typical of older star-forming or starburst regions. (4) The $\approx 2$ mag drop between the $R$ and the $I$ bands can be explained by

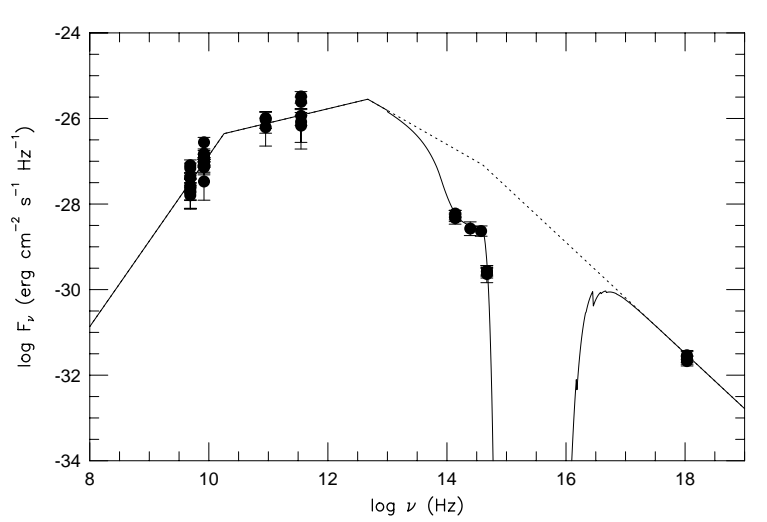

Fig. 2. The radio through X-ray spectrum of the afterglow of GRB 980329. All measurements have been scaled to a common time, approximately three days after the GRB. The solid curve is the best fit spectrum for an isotropic fireball that expands into a homogeneous external medium, extincted by dust at a redshift of $z=3.5$. The dotted curve is the un-extincted spectrum

the Ly- $\alpha$ forest if the burst redshift is $z \approx 5$ (Fruchter 1999), by the far-ultraviolet non-linear component of the extinction curve if $3 \lesssim z \lesssim 5$, and by the $2175 \AA$ bump if $z \approx 2$; other redshifts are not consistent with these data, given this general model. Djorgovski et al. (1999) report that $z<3.9$ based upon the non-detection of the Ly- $\alpha$ forest in a Keck II spectrum of the host galaxy. (5) Assuming a redshift of $z=3.5$ for illustrative purposes, using the observed breaks in the intrinsic spectrum, and solving for the physical properties of the fireball (see, e.g., Wijers \& Galama 1998), we find that the energy of the fireball per unit solid angle is $\mathcal{E} \sim 10^{52} / 4 \pi \mathrm{erg} \mathrm{sr}^{-1}$ if $\Omega_{\mathrm{m}}=0.3$ and $\Omega_{\Lambda}=0.7$. (6) Similarly, we find that the density of the external medium into which this fireball expands is $n \sim$ $10^{3} \mathrm{~cm}^{-3}$ if $\Omega_{\mathrm{m}}=0.3$ and $\Omega_{\Lambda}=0.7$. This density suggests that GRB 980329 occurred in a molecular cloud, which is consistent with the fact that the observed extinction features are characteristic of star-forming regions.

\section{References}

Cardelli J.A., Clayton G.C., Mathis J.S., 1989, ApJ 345, 245

Castander F.J., Lamb D.Q., 1998, in Gamma-Ray Bursts, Meegan C.A., Preece R.D., Koshut T.M. (eds.). New York: AIP, p. 520

Castander F.J., Lamb D.Q., 1999a, ApJ (submitted)

Castander F.J., Lamb D.Q., 1999b, ApJ (submitted)

Djorgovski S.G., et al., 1999, invited talk at the Santa Barbara Workshop "Gamma-Ray Bursts and their Afterglows"

Fioc M., Rocca-Volmerange B., 1997, A\&A 326, 950

Fitzpatrick E.L., Massa D., 1988, ApJ 328, 734

Fruchter A.S., 1998, ApJL (in press)

Fruchter A.S., et al., 1998, ApJ (submitted)

González R.A., Fruchter A.S., Dirsch B., 1999, ApJ (submitted)

Kulkarni S.R., et al., 1997, Nat 393, 35

Sari R., Piran T., Narayan R., 1998, ApJ 497, L17

Tonry J.L., et al., 1997, IAU Circular No. 6620

Wijers R.A.M.J., Galama T.J., 1998, ApJ (submitted) 